\title{
artigo
}

Silvestre, M.M.; Pereira, F.R.; Pennacchi, R.A.; Macêdo Junior, A.G.;

Fatores históricos e de vulnerabilidades associados a transmissão da hanseníase no Brasil

\section{Fatores históricos e de vulnerabilidades associados a transmissão da hanseníase no Brasil}

\author{
Historical and vulnerability factors associated with transmission of leprosy in Brazil \\ Factores históricos y de vulnerabilidad asociados con la transmisión de la lepra en Brasil
}

\begin{abstract}
RESUMO
Objetivo: 0 estudo teve como objetivo descrever o papel da história de políticas públicas e de fatores de vulnerabilidade ainda associados à transmissão da hanseníase no Brasil. Método: Trata-se de um estudo descritivo através de revisão bibliográfica a partir de busca de artigos dos últimos 20 anos em bases de dados. Resultados: Verificou-se que fatores biológicos, clínicos, socioeconômicos e políticos ainda encontram-se sendo relevantes para manutenção de elevados índices e de incapacidades físicas associados à hanseníase no país. Conclusão: Os autores recomendam para atingir os objetivos propostos pela Organização Mundial de Saúde de eliminação da hanseníase, uma abordagem multiprofissional, com atores das áreas de saúde, humanas e exatas, em ações integradas entre a atenção básica, vigilância em saúde e gestores de políticas públicas.
\end{abstract}

DESCRITORES: Hanseníase; Lepra; Política; Vulnerabilidade; Transmissão.

\section{ABSTRACT}

Objective: The study aimed to describe the role of the history of public policies and of vulnerability factors still associated with leprosy transmission in Brazil. Method: This is a descriptive study through a bibliographic review based on the search for articles from the last 20 years in databases. Results: It was found that biological, clinical, socioeconomic and political factors are still relevant to maintaining high rates and physical disabilities associated with leprosy in the country. Conclusion: The authors recommend to achieve the objectives proposed by the World Health Organization for the elimination of leprosy, a multiprofessional approach, with actors from the areas of health, human and exact, in integrated actions between primary care, health surveillance and public policy managers.

DESCRIPTORS: Leprosy; Policy; Vulnerability; Streaming.

\section{RESUMEN}

Objetivo: El estudio tuvo como objetivo describir el papel de la historia de las políticas públicas y de los factores de vulnerabilidad aún asociados a la transmisión de la lepra en Brasil. Método: Se trata de un estudio descriptivo mediante revisión bibliográfica a partir de la búsqueda de artículos de los últimos 20 años en bases de datos.. Resultados: Se encontró que los factores biológicos, clínicos, socioeconómicos y políticos siguen siendo relevantes para mantener altas tasas y discapacidades físicas asociadas a la lepra en el país. Conclusión: Los autores recomiendan lograr los objetivos propuestos por la Organización Mundial de la Salud para la eliminación de la lepra, un enfoque multiprofesional, con actores de las áreas de salud, humana y exacta, en acciones integradas entre atención primaria, vigilancia de la salud y gestores de políticas públicas.

DESCRIPTORES: Lepra; Política; Vulnerabilidad; Transmisión.

RECEBIDO EM: 06/01/2021 APROVADO EM: 18/02/2021

\section{Mariana Montalvão Silvestre}

Graduada em medicina pela Universidade Federal do Oeste da Bahia. Colaboradora no grupo de pesquisa Doenças Infecciosas e Parasitárias (DIP).

ORCID: 0000-0003-0724-9055

\section{Fernanda Rebouças Pereira}

Discente do curso de graduação em medicina da Universidade Federal do Oeste da Bahia. Colaboradora no grupo de pesquisa Doenças Infecciosas e Parasitárias (DIP).

ORCID: 0000-0003-3652-8635 


\section{Rafael Attiê Pennacchi}

Discente do curso de graduação em medicina da Universidade Federal do Oeste da Bahia.

ORCID: 0000-0003-4983-9475

\section{Arlindo Gomes de Macêdo Junior}

Graduado em Biomedicina na Universidade Estadual de Santa Cruz - UESC. Mestrado e Doutorado em Imunologia e Parasitologia Aplicadas na Universidade Federal de Uberlândia - UFU. Atualmente é Professor Adjunto C da Universidade Federal do Oeste da Bahia e coordenador do grupo de pesquisa Doenças Infecciosas e Parasitárias (DIP).

ORCID: 0000-0002-3731-3428

\section{INTRODUÇÃO}

A hanseníase é uma doença altamente incapacitante e ainda estigmatizada pelo seu histórico dos séculos passados $^{1}$. Em todo o mundo, mais de 200.000 novos casos são detectados anualmente, com a Índia, Brasil e a Indonésia representando cerca de $80 \%$ de todos os novos casos ${ }^{2}$.

A hanseníase de apresenta com um quadro clínico que varia de pessoa para pessoa, tendo como principais manifestações: a presença de uma ou mais manchas hipocrômicas, hipercrômicas ou eritematosas; parestesias no local das lesões; pápulas, tubérculos e nódulos; espessamento dos nervos e dor, perda de sensibilidade e/ ou força nas áreas dos nervos afetados ${ }^{1,3,4}$.

O Brasil é o país com maior incidência e prevalência de hanseníase no mundo e o único entre os 35 países da Organização Pan-Americana de Saúde (OPAS) que ainda não a eliminou como problema de saúde pública 5 . Em 2018 foram registrados 28.660 novos casos no Brasil, 13,73\% de todos os novos casos detectados no mundo e 92,6\% dos casos detectados nas Américas. Desses, aproximadamente $8,5 \%$ foram detectados com grau 2 de deformidade ${ }^{6}$.

Devido à grande extensão do Brasil, sabe-se que a hanseníase se distribui de forma heterogênea entre os estados e municípios, com regiões com padrões de saúde semelhantes aos dos países desenvolvidos e regiôes com índices de mortalidade semelhantes aos dos países mais pobres do hemisfério sull,8.

Somando ao tamanho territorial, diferentes fatores estão associados à transmissão da hanseníase no Brasil, sendo importante a realização de estudos para caracterização dos perfis de vulnerabilidades envolvidas na sua transmissão e do histórico de políticas públicas do seu enfrentamento no país.

\section{METODOLOGIA}

$\mathrm{O}$ artigo trata-se de um estudo descritivo através de revisão bibliográfica. A pesquisa foi realizada nos bancos de dados Scielo (Scientific Electronic Libray Online) e Pubmed utilizando os descritores hanseníase AND política AND Brasil; hanseníase AND transmissão AND Brasil, em português e inglês. Como critério de inclusão foi estabelecido que os artigos deveriam ser completos, publicados nos últimos 20 anos em revistas indexadas, apresentar discussões sobre fatores envolvidos na vulnerabilidade e controle da hanseníase no Brasil e ser escrito em idiomas português ou inglês. Durante o processo de leitura realizou-se apontamento dos principais fatores para responder a questão norteadora: quais os fatores de vulnerabilidades ainda estão envolvidos com a transmissão da hanseníase no Brasil? Para avaliar o papel das políticas envolvidas no cenário da hanseníase no país, levantou-se historicamente as principais ações públicas de enfrentamento durante os últimos anos

\section{RESULTADOS}

Após uma avaliação crítica pelos autores, os dados encontrados nos apontamentos foram reunidos e as principais informações foram sintetizadas em formato de texto. Conforme análise da literatura, os dados serão apresentados conforme os principais fatores biológicos, clínicos e epidemiológicos, geográficos, socioeconômicos e políticos envolvidos na continuidade da prevalência da hanseníase no Brasil.

\section{Fatores biológicos}

Considerando a relação parasito-hospedeiro, apenas $5 \%$ dos indivíduos em contato com o patógeno são suscetíveis ao quadro da hanseníase. Como a variabilidade do M. leprae é baixa, o perfil genético e de polarização da resposta imune do hospedeiro são cruciais no combate ao patógeno $9,10,11$. Alterações nos genes de PARK2, IL-10 e LTA e nas variações codificantes de TLR1 e SLC11A1 são exemplos de associação com a leprosy per se por reduzirem a produção de IL-6 e CCL2 em macrófagos ${ }^{12}$.

Em 2009, o genome-wide association of leprosy (GWAS), descreveu os principais genes envolvidos na resposta para a hanseníase ${ }^{13}$, sendo citado entre os principais a via NOD2 e LACC1. O receptor NOD2 reconhece o dipeptideo muramil e sinaliza para a produção de citocinas pró-inflamatórias necessárias para a atividade antimicrobiana $^{13,14}$. Já o LACC1 interage diretamente com NOD2, produzindo espécies reativas ao oxigênio e produção de citocinas pró-inflamatorias. Polimorfismos em LACC1 constituem fator de risco devido à redução da sinalização via LACC1-NOD215. Outro exemplo é o gene HLA-A*28, relacionado com a forma clínica dimorfa e virchowiana ${ }^{16}$. Outros polimorfismos associados ao início da polarização para as formas clínicas ainda estão sendo pesquisados ${ }^{17}$. Relacionado ao patógeno, alterações no tetratricopeptídeos (TTC) da M. leprae podem estar contribuindo para a resistência à poliquimioterapia $^{18,19}$.

\section{Fatores clínicos e epidemiológicos}

A hanseníase é mais prevalente nos homens do que em mulheres e estes apresentam um risco maior de serem diagnos- 
ticados com o grau dois de incapacidade física $^{20}$. Entre os fatores envolvidos encontram-se a demora para procurar os serviços de saúde e a carência de mais programas direcionados à saúde do homem, sendo que a chance de não aderirem ao tratamento é três vezes maior do que as mulheres ${ }^{20,21}$.

Ao traçar o perfil epidemiológico no Brasil, Veloso et al., 2018, revelou a forma indeterminada como a menos frequente, sendo justificada pela dificuldade de diagnóstico por apresentar baixo número de lesões ${ }^{22}$.

No Brasil a faixa etária mais acometida é a economicamente ativa, com um impacto indireto sobre a economia, principalmente quando o diagnóstico é tardio e o indivíduo já apresenta incapacidades físicas $^{22}$. A não realização da baciloscopia ou a sua não notificação, revela ser ainda um exame negligenciado no diagnóstico e classificação da hanseníase ${ }^{8,23}$. A OMS instituiu que para alcançar as metas para 2020 deve-se concentrar esforços na detecção oportuna de casos antes das incapacidades virem a acontecer, principalmente com exame de coletividades ${ }^{24}$.

O tratamento inadequado é um importante fator de risco para transmissão da doença, visto que os pacientes multibacilares continuaram transmitindo a bactéria. A recidiva da doença pode ocorrer anos após a poliquimioterapia irregular e/ou ineficaz, com possível surgimento de cepas resistentes ao tratamento, constituindo um risco para a sociedade ${ }^{1}$.

Os episódios reacionais, que podem aparecer antes, durante ou depois do tratamento, são encontrados em 30 a 50\% dos indivíduos. Em 2015, em aproximadamente 5,5\% dos novos casos no Brasil foram detectados grau 2 de deformidade, com lesões graves que comprometem a funcionalidade do membro ou órgão acometido e $67,86 \%$ eram multibacilares ${ }^{2}$.

O grau de incapacidade física está relacionado indiretamente com a falta de informação da população sobre a clínica da doença, com o tempo entre a doença e o seu diagnóstico e com a falta de acesso a serviços com profissionais capacitados para detectar a hanseníase de forma precoce. $5,7 \%$ das pessoas que descobrem ter hanseníase no Brasil, já apresentam lesões sensitivas e/ou motoras que poderiam ter sido evitadas ${ }^{25}$. Assim, o grau de incapacidade é um indicador para avaliação da efetividade do diagnóstico precoce e da adesão das pessoas ao tratamento ${ }^{26}$. Recomenda-se que gestores de saúde estejam atentos a possíveis aumentos de multibacilares e no número de abandono do tratamento e de incapacidades físicas.

\section{Fatores sociais e econômicos}

As condições socioeconômicas têm grande influência na distribuição e propagação da hanseníase, com estreita relação com condições precárias de habitação, baixa escolaridade e renda e com os movimentos migratórios ${ }^{27,28}$.

A transmissão domiciliar da hanseníase está associada a um risco 2,9 a 5,0 vezes maior quando já possui um caso na família ${ }^{29}$. Como a transmissão se dá pelas vias aéreas através do contato íntimo, o contato prolongado com um indivíduo com elevado números de bacilos e não tratado predispõem o contágio ${ }^{30}$. A moradia com grande quantidade de pessoas em um mesmo ambiente é um fator de vulnerabilidade para a manutenção da cadeia de transmissão do bacilo ${ }^{1,31,32}$.

$\mathrm{O}$ alto índice de casos em indivíduos com baixa escolaridade é um marcador indireto de autocuidado precário ${ }^{1,29,32}$. A educação formal propicia o conhecimento sobre as necessidades de saúde, busca de assistência médica e maior acesso aos serviços de saúde ${ }^{33}$. O alto grau de escolaridade já foi apontado como fator de proteção para a hanseníase e para completar o esquema poliquimioterapia ${ }^{32,34}$.

A hanseníase também possui profunda relação com movimentos migratórios, uma vez que, facilitam a propagação da doença ${ }^{27}$, sendo importante ações integradas na atenção básica para um rastreio de possíveis casos de infecções, acolhimento e acompanhamento de famílias provenientes de efeitos migratórios em seus territórios.

\section{Fatores geográficos}

Com a grande extensão do Brasil, a hanseníase se distribui de forma heterogênea, com regiões com padróes de saúde semelhantes aos dos países desenvolvidos e regiões com índices de mortalidade semelhantes aos dos países mais pobres do hemisfério sul $^{8,35}$. O maior coeficiente de prevalência está no Centro-Oeste, Norte e Nordeste, tidos tradicionalmente como regióes socioeconomicamente desfavoráveis ${ }^{36}$. Já a região Sul e Sudeste possui um índice de prevalência menor e taxa de cura elevada ${ }^{8}$.

Nesse contexto, o geoprocessamento, definido como um conjunto de técnicas matemáticas e computacionais que são utilizadas para produzir materiais cartográficos ${ }^{31}$, permite a visualização da distribuição de doenças e agravos na coletividade. A própria OPAS recomenda que países com grande extensão territorial façam uso da análise espacial para que os serviços de saúde se reorganizem para atender as necessidades da população ${ }^{37}$. Essa análise é usada para identificar áreas com altas incidências relacionadas com as desigualdades sociais, ajuda a melhorar as medidas de vigilância epidemiológica e a elucidar a tendência espacial que a hanseníase segue de acordo com a realidade de cada local 28,36,38.

Atualmente a qualidade da notificação de casos influencia no geoprocessamento e análise de dados de territorialização. Ao avaliar a qualidade dos dados em alguns locais referentes à hanseníase, parte das notificações no SINAN estão incompletas ou divergem entre suas próprias informações ${ }^{39}$. Melhorias nas notificações são importantes para traçar perfis epidemiológicos e espaciais da hanseníase, possibilitando planejamento de políticas públicas voltadas para as reais necessidades da população.

\section{Fatores históricos e políticos}

As políticas públicas de eliminação da hanseníase são elaboradas em todo o mundo. Em 1991, a OMS estabeleceu como meta reduzir o coeficiente de prevalência de 1 para cada 10.000 habitantes até $2000^{40}$. A partir de 1998 foram descentralizadas as ações de controle para a atenção básica ${ }^{41,42}$. Em 2004, com o Programa Nacional de Eli- 
minação da Hanseníase (PNEH), ocorreu o redirecionamento das políticas de atenção à hanseníase aos municípios. Isso possibilitou a visibilidade da distribuição da hanseníase no país com atividades baseadas na realidade de cada local ${ }^{42}$.

Em 2005, sem sucesso em eliminar a hanseníase, o Brasil lançou o Plano Nacional para Eliminação da Hanseníase 2006-2010, com o objetivo da descentralização da Atenção Básica, o compromisso de governos estaduais e municipais para se encarregarem da oferta de emprego para os indivíduos vivendo com a hanseníase, a com garantia de poliquimioterapia e capacitação dos profissionais de saúde ${ }^{1}$.

Já em 2010, foi publicada a portaria $\mathrm{n}^{\circ} 3.125$ que aprovou as Diretrizes para Vigilância, Atenção e Controle da Hanseníase, as quais preconizavam ações para toda a rede de Atenção Primária do SUS e garantia do atendimento especializado na rede ambulatorial e hospitalar sempre que necessário, fortalecendo assim a atenção integral e a promoção de saúde no Brasil ${ }^{43}$.

No entanto, em 2011 o Ministério da Saúde considerou os resultados insuficientes e incompatíveis com a capacidade do SUS. Tentando resolver esse problema, foi criada a Coordenação Geral de Hanseníase e Doenças em eliminação que tinha como principal objetivo fortalecer a resposta para a hanseníase e outras doenças infecciosas endêmicas na população de baixa renda e consideradas negligenciadas ${ }^{1}$.

A partir de então, foi lançado o "Plano integrado de ações estratégicas de eliminação da hanseníase, filariose, esquistossomose e oncocercose como problema de saúde pública, tracoma como causa de cegueira e controle das geohelmintíases" a ser realizado no período de 2011 a $2015^{1,35}$. O plano tinha como um dos principais objetivos garantir o acesso da população mais pobre aos serviços de saúde, otimizando os recursos para o combate dessas doenças. E em 2016, essa mesma equipe lançou as "Diretrizes para vigilância, atenção e eliminação da hanseníase como problema de saúde pública" que objetivava, além de eliminar a doença, uniformizar o atendimento da pessoa com hanseníase e padronizar os pro- cedimentos de vigilância válido para todo o território nacional ${ }^{18,43}$.

Já mais recente, a OMS lança em 2016 a Estratégia de Eliminação da Hanseníase 2016-2020, com objetivo de interrupção global da transmissão ou sua eliminação e redução de deficiências de grau $2 \mathrm{em}$ casos recém-detectados para abaixo de 1 por milhão de população em nível global até $2020^{2}$. A estratégia se apoiou em fortalecer o controle, a coordenação e a parceria do governo em fornecer recursos suficientes e subsidiar pesquisas, em combater a hanseníase e suas complicações, no combate da discriminação e na promoção da inclusão social ${ }^{2}$.

\section{No mais, é de}

extrema importância

o fortalecimento

de estratégias para

evitar o abandono à

poliquimioterapia.

Todos os planos e estratégias de eliminação da hanseníase instituídos no Brasil desde 1991, com introdução da poliquimioterapia e o aumento da detecção de novos casos foram a causa da diminuição de cerca de $90 \%$ do número global de casos nas últimas duas décadas ${ }^{1}$. A redução da taxa de detecção dos casos novos ocorreram principalmente na região sul e sudeste, em que a maioria dos municípios conseguiram controlar a hanseníase ${ }^{8}$. Porém, nas regióes norte, centro-oeste e nor- deste, a redução do coeficiente se mostra de forma lenta e insuficiente ${ }^{44}$.

\section{DISCUSSÃO}

Diantes dos fatores de vulnerabilidade encontrados para a prevalência da hanseníase no Brasil, as avaliações da implantação de programas municipais de controle da hanseníase e da Estratégia de Saúde da Família (ESF) quanto ao diagnóstico, classificação e tratamento da hanseníase ainda são de grande relevância para o alcance das metas instituídas pela OMS.

Ações em saúde, incluindo a busca ativa para um diagnóstico precoce e estabelecimento de tratamento adequado, consistem em importantes estratégias de enfrentamento e que podem ser realizadas para eliminar o surgimento de novos casos e agravos associados à hanseníase45,46. Em escolas dos municípios poderão ser realizadas ações de buscas de casos em menores de 15 anos $^{47,48}$.

Outro aspecto importante para o enfrentamento das questões de vulnerabilidades é a necessidade de atividades de capacitação dos profissionais das ESFs quanto às ações básicas que podem ser realizadas visando o controle da hanseníase e a prevenção de incapacidades ${ }^{49}$.

A conscientização dos profissionais de saúde para um maior cuidado no preenchimento das informações nas fichas de notificações também consiste em estratégia importante para as políticas de vigilâncias e futuros estudos retrospectivos situacionais em diferentes territórios.

No mais, é de extrema importância o fortalecimento de estratégias para evitar o abandono à poliquimioterapia. Levando em consideração as particularidades de cada indivíduo, localidade e do serviço de saúde, existem uma pluralidade de fatores envolvidos com a interrupção e abandono do tratamento ${ }^{50,51}$. Esses fatores devem ser conhecidos e ações específicas para cada necessidade local devem ser realizadas. Ações como essas poderão prevenir a transmissão, progressão da doença e o desenvolvimento de incapacidades físicas no indivíduo infectado. 


\section{CONCLUSÃO}

Nos últimos anos, o Brasil avançou em políticas públicas e ações de combate para novos casos e diminuição do número de agravos relacionados à hanseníase. No entanto, o país ainda encontra desafios para eliminar a sua transmissão. $\mathrm{O}$ cenário epidemiológico do país evidencia a necessidade de integração entre profissionais da saúde, humanas e exatas, de forma multiprofissional, para atuar na atenção básica e na vigilância em saúde. Essa integração será importante para compreender a situação da hanseníase no país, bem como no planejamento em saúde para seu enfrentamento.

\section{REFERÊNCIAS}

1. Alves ED, Ferreira, TL, Ferreira, IN. Hanseníase - Avanços e Desafios. 1.ed. Brasília: Universidade de Brasília; 2014.

2. Organização Mundial da Saúde (OMS). Estratégia Global para Hanseniase 2016-2020: Aceleração rumo a um mundo sem Hanseníase. OMS, 2016.

3. Ministério da Saúde (BR). Guia para o controle da hanseníase. Brasilía: Ministério da Saúde, 2002.

4. Burns, T. Rook's Textbook of Dermatology. 8. ed. Leicester, Reino Unido: Willey-Blackwell, 2013

5. Organização Pan-Americana de Saúde (OPAS). Detecção proativa e precoce é essencial para acabar com deficiências relacionadas à hanseníase em crianças. Brasília: OPAS, 2017.

6. Ministério da Saúde do Brasil (BR). Boletim epidemiológico de Hanseníase. Brasília: Ministério da Saúde, 2020.

7. Ministério da Saúde (BR). Plano integrado de ações estratégicas de eliminação da hanseníase, filariose, esquistossomose e oncocercose como problema de saúde pública, tracoma como causa de cegueira e controle das geohelmintíases. Brasília: Ministério da Saúde, 2013.

8. Ribeiro MDA, Silva JCA, Oliveira SB. Estudo epidemiológico da hanseníase no Brasil: reflexão sobre as metas de eliminação. Rev Panam Salud Publica. 2018; 42: e42.

9. Mendonça Vanessa Amaral, Costa Rosane Dias, Melo Gustavo Eustáquio Brito Alvim de, Antunes Carlos Maurício, Teixeira Antonio Lúcio. Imunologia da hanseníase. An. Bras. Dermatol. 2008; 83(4): 343-350.

10. Lastória, JC, Abreu MAMM. Hanseníase: diagnóstico e tratamento. Diagn Tratamento. 2012; 17(4): 173-179.

11. Lazaro FP, Werneck RI, Mackert CC, Cobat A, Prevedello FC, Pimentel RP, Macedo GM, Eleuterio MA, Vilar G, Abel L, Xavier MB, Alcais A, Mira MT. A major gene controls leprosy susceptibility in a hyperendemic isolated population from north of Brazil. J Infect Dis. 2010; 201: 1598-1605.

12. Léséleuc L, Orlova M, Cobat A, Girard M, Huong NT, Ba NN, et al. PARK2 Mediates Interleukin 6 and Monocyte Chemoattractant Protein 1 Production by Human Macrophages. PLOS Neglected Tropical Diseases. 2013; 7(1): e2015

13. Zhang FR, Huang W, Chen SM, Sun LD, Liu H, Cui Y, et al. Genomewide association study of leprosy. N Engl J Med. 2009; 361(27): 2609-2618

14. Toledo Pinto TG, Batista-Silva LR, Medeiros RCA, Lara FA, Moraes MO. Type I Interferons, Autophagy and Host Metabolism in Leprosy. Front Immunol. 2018; 9: 806.

15. Lahiri A, Hedle, M, Yan, J. Human LACC1 increases innate receptor-induced responses and a LACC1 disease-risk variant modulates these outcomes. Nat Commun. 2017; 8: 15614

16. Aguilar-Medina M, Escamilla-Tilch M, Frías-Castro LO, Romero-Quintana G, Estrada-García I, Estrada-Parra S, et al. HLA Alleles are Genetic Markers for Susceptibility and Resistance towards Leprosy in a Mexican Mestizo Population. Ann Hum Genet. 2017; 81(1): 35-40.

17. Gaschignard J, Grant AV, Thuc NV, Orlova M, Cobat A, Huong NT, et al. Pauci- and Multibacillary Leprosy: Two Distinct, Genetically Neglected Diseases. PLOS Neglected Tropical Diseases 2016; 10(5): e0004345

18. Reja AHH, De A, Patra PK, Biswas S, Duttagupta U, Sil A, et al. Genomic Reduction at TTC Repeats in the Bacterial Genome of Treated Cases of Hansen's Disease: A Possible Survival Mechanism of Mycobacterium leprae. Indian J Dermatol. 2018; 63(6): 449-454

19. Matsuoka M, Zhang L, Budiawan T, Saeki K, Izumi S. Genotyping of Mycobacterium leprae on the basis of the polymorphism of TTC repeats for analysis of leprosy transmission. J Clin Microbiol. 2004; 42(2): 741-745

20. Romão ER, Mazzoni AM. Perfil epidemiológico da hanseníase no município de Guarulhos, SP. Revista de Epidemiologia e Controle de Infecção. 2013; 3(1): 22-27.

21. Monteiro MJ, Andrade SSC, Santana EMF, Peixoto BV, Nogueira JA, Soares MJGO. Perfil Epidemiológico De Casos De Hanseníase Em Um Estado Do Nordeste Brasileiro. Revista Brasileira Ciências da Saúde - USCS. 2017; 15(54): 21-28.

22. Velôso DS, Melo CBS, Santos TLB, Nascimento JP, Costa EF, Carvalho FA. Perfil Clínico Epidemiológico da Hanseníase: Uma Revisão Integrativa. 2017; 10(1): 1464-1471.

23. Neves TV, Valentim IM, Reis IB, Souza EB, Diniz AP Rocha ESD, et al. Informações do SINAN e de prontuários de unidades de saúde da familía acerca de incapacidades físicas decorrentes da hanseníase em Palmas, Tocantins. Revista Eletrônica Gestão \& Saúde. 2015; 6(3): 2460-70.

24. Ministério da Saúde (BR). Casos novos de hanseníase por estados e regiões. Brasília, Ministério da Saúde, 2016.

25. Arantes Cíntia Kazue, Garcia Maria Luiza Rufino, Filipe Mariana Scombatti, Nardi Susilene Maria Tonelli, Paschoal Vânia Del'Arco. Avaliação dos serviços de saúde em relação ao diagnóstico precoce da hanseníase. Epidemiol. Serv. Saúde [Internet]. 2010; 19(2): 155-164.

26. Alves Cinthia Janine Meira, Barreto Jaison Antônio, Fogagnolo Leticia, Contin Leticia Arsie, Nassif Priscila Wolf. Avaliação do grau de incapacidade dos pacientes com diagnóstico de han- 


\section{REFERÊNCIAS}

seníase em serviço de dermatologia do estado de São Paulo. Rev. Soc. Bras. Med. Trop. 2010 ; 43( 4 ): 460-461

27. Amaral Evaldo Pinheiro, Lana Francisco Carlos Félix. Análise espacial da Hanseníase na microrregião de Almenara, MG, Brasil. Rev. bras. enferm. [Internet]. 2008; 61( spe ): 701-707.

28. Imbiriba Elsia Nascimento Belo, Silva Neto Antônio Levino da, Souza Wayner Vieira de, Pedrosa Valderiza, Cunha Maria da Graça, Garnelo Luiza. Desigualdade social, crescimento urbano e hanseníase em Manaus: abordagem espacial. Rev. Saúde Pública . $2009 ; 43(4): 656-665$

29. Santos Andréia Soprani dos, Castro Denise Silveira de, Falqueto Aloísio. Fatores de risco para transmissão da Hanseníase. Rev. bras. enferm. [Internet]. 2008; 61( spe ): 738-743.

30. Gonçalves KS. Indicadores Epidemiológicos e Análise Espacial dos Casos Novos de Hanseníase no Município de Serra: Tendência Temporal e Efetividade do Programa de Controle da Doença [dissertação]. Vitoria: Universidade Federal do Espírito Santo, 2014.

31. Gracie Renata, Peixoto Julia Novaes de Barros, Soares Fabiane Bertoni dos Reis, Hacker Mariana de Andrea Vilas-Boas. Análise da distribuição geográfica dos casos de hanseníase. Rio de Janeiro, 2001 a 2012. Ciênc. saúde coletiva [Internet]. 2017; 22( 5 ): 1695-1704.

32. Júnior AFR, Vieira MA, Caldeira AP. Perfil epidemiológico da hanseníase em uma cidade endêmica no Norte de Minas Gerais. Revista Brasileira de Clinica Médica. 2012; 10(4): 272-277.

33. Franco-Paredes C, Marcos LA, Henao-Martínez AF, Rodríguez-Morales AJ, Villamil-Gómez WE, Gotuzzo E, Bonifaz A. Cutaneous Mycobacterial Infections. Clin Microbiol Rev. 2018 32(1): e00069-18.

34. Gonçalves-Brito KK, Andrade SSC, Diniz IV, Matos SDO, OIiveira SHS, Oliveira MJG. Caracterização Dos Casos De Hanseníase Diagnosticados Através Do Exame De Contato. Journal of Nursing UFPE / Revista de Enfermagem UFPE. 2016; 10(2): 435-441.

35. Ministério da Saúde (BR). Plano integrado de ações estratégicas de eliminação da hanseníase, filariose, esquistossomose e oncocercose como problema de saúde pública, tracoma como causa de cegueira e controle das geohelmintíases. Brasília: Ministério da Saúde, 2013.

36. Ribeiro MA, Albuquerque IZMN, Vasconcelos MIO, Dias LKS, Cavalcante ASP. Geoprocessamento Em Saúde Como Tecnologia De Análise E Monitoramento Da Hanseníase No Município De Sobral-Ceará. Revista Baiana de Saúde Pública. 2018; 41(2): 451-465.

37. Organização Pan-Americana da Saúde. Uso de los Sistemas de Información Geográfica em Epidemiologia (SIG-EPI). Boletim Epidemiológico. 1996; 17(1).

38. Nardi S, Pedro H, Seixas L, Amorim K, Cunha, R, Freitas A, Angelo L, Paschoal V. Comportamento da endemia da hanseníase com base nos sinais cardinais. Arquivos De Ciências Da Saúde.
2017; 24(2): 82-87.

39. Galvão PRS, Ferreira AT, Maciel MGG, Almeida RP, Hinders D, Schreuder PA, et al. Uma avaliação do sistema de informação SINAN usado no Programa de Controle de Hanseníase no estado do Pernambuco, Brasil. Cadernos de Saúde Coletiva. 2009; 17(1): 87-102.

40. Ministério da Saúde (BR). Controle da Hanseníase na Atenção Básica. Brasília: Ministério da Saúde, 2001.

41. Borba SMLS. Vigilância epidemiológica da hanseníase na atenção básica: o caso do município de Itaboraí, região metropolitana do Rio de Janeiro [dissertação]. Rio de Janeiro: Fundação Oswaldo Cruz, 2015.

42. Costa MS, Junior PCB, Moura JPG, Pantoja PVN. Policies for leprosy: the evolution of management in health. Revista Enfermagem Digital Cuidado e Promoção da Saúde. 2015; 1(2): 99103.

43. Ministério da Saúde (BR). Diretrizes Para Vigilância, Ação e Controle da Hanseníase. Brasília: Ministério da Saúde, 2010.

44. Leal Danielle Rodrigues, Cazarin Gisele, Bezerra Luciana Caroline Albuquerque, Albuquerque Ana Coelho de, Felisberto Eronildo. Programa de Controle da Hanseníase: uma avaliação da implantação no nível distrital. Saúde debate [Internet]. 2017; 41( spe ): 209-228.

45. Rodrigues, Elaine Fernanda, Márcio José Trovarelli, and Nágila Garcia Galan de Oliveira. Busca ativa de hanseníase numa comunidade de trabalhadores rurais em uma usina de cana-deaçucar. 2009: 44-44.

46. Pereira Elizane Viana Eduardo, Nogueira Lidya Tolstenko, Machado Herion Alves da Silva, Lima Lana Andrade Napoleão, Ramos Clóvis Henrique Mauriz. Perfil epidemiológico da hanseníase no município de Teresina, no período de 2001-2008. An. Bras. Dermatol. [Internet]. 2011; 86(2): 235-240.

47. Moura Luiza Taciana Rodrigues de, Fernandes Tânia Rita Moreno de Oliveira, Bastos Lívia Dias Mangueira, Luna Igara Cavalcanti Feitosa, Machado Lara Barreto. Hanseníase em menores de 15 anos na cidade de Juazeiro-BA. Hansenol. Int. 2012 Jun; 37(1): 45-50.

48.Schneider Priscila Barros, Freitas Bruna Hinnah Borges Martins de. Tendência da hanseníase em menores de 15 anos no Brasil, 2001-2016. Cad. Saúde Pública [Internet]. 2018; 34(3): e00101817.

49.Oliveira CM, Linhares MSC, Neto FRGX, Mendes IMVP, Kerr LRFS. Conhecimento e práticas dos Agentes Comunitários de Saúde sobre hanseníase em um município hiperendêmico. Saúde em Revista. 2018; 18(48): 39-50.

50. Girão RJ, Soares NL, Pinheiro JV, Oliveira Gda P, de Carvalho SM, de Abreu LC, Valenti VE, Fonseca FL. Leprosy treatment dropout: a systematic review. Int Arch Med. 2013; 6(1): 34.

51. Saraswat N, Agarwal R, Chopra A, Kumar S, Dhillon A. Assessment of Factors Responsible for Dropout to Multi Drug Therapy for Leprosy. Indian Journal of Leprosy. 2019; 91(3): 225-232 